\title{
MeDevice: A Mobile - Based Diagnosis of Common Human Illnesses using Neuro - Fuzzy Expert System
}

\author{
Johaira U. Lidasan \\ Notre Dame University, Cotabato City, 9600, Philippines \\ E-mail: tequierophardz@gmail.com \\ Martina P. Tagacay \\ Notre Dame University, Cotabato City, 9600, Philippines \\ E-mail: mptagacay0130@gmail.com
}

Received: 25 April 2019; Accepted: 23 May 2019; Published: 08 August 2019

\begin{abstract}
Fever is a sign that the body is trying to fight infection. It is usually accompanied by various sicknesses or symptoms that signal another illness or disease. Diagnosing it ahead of time is essential because it has to do with human life and to determine what to do to get well. MeDevice is a mobile-based application that runs in Android devices that allows the user to enter the levels of his/her symptoms and diagnoses the disease either as influenza, dengue, chicken pox, malaria, typhoid fever, measles, Hepatitis A and pneumonia together with its details and its first aid treatment. It aims at providing an efficient decision support platform to aid people with fever in diagnosing their disease and whether or not to seek medical attention especially in developing countries like the Philippines. This application is engineered with the knowledge base and the inference method of fuzzy logic and expert system with the help of Gradient Descent optimization algorithm and back propagation neural network to achieve the optimum value of the error rate. This is essential to provide the application with a high accuracy rate which shows during the conduct of testing of the application.
\end{abstract}

Index Terms-Fever, Neuro-fuzzy expert, hybrid system, Gradient descent, optimization algorithm.

\section{INTRODUCTION}

Fever is an increase in body temperature. Usually, this is the result when the body is trying to fight an infection. It is usually accompanied by various sicknesses or symptoms that most often than not, signals another illness or diseases. There are a lot of diseases that are associated with or have been called fever that affects almost all human beings [1]. Therefore, diagnosing the disease associated with fever is essential because it has to do with human life and to determine what to do to get well.

The only problem is people tend to avoid seeking medical help. Based on the conducted researches [2, 3], they prefer to stay at home and wait for their illness to improve over time especially in developing countries. This is not good since it could make the illness worst and/or infect other people. Therefore, having an application that will help the people in diagnosing their disease is of great advantage.

Many studies have been conducted to diagnose diseases with the help of Artificial Intelligence such as diagnosing diabetes, depression, typhoid fever, leukemia etc. Artificial intelligence is a way of making a computer in a similar manner the intelligent humans think [4]. It has two goals: one is to create intelligent systems that emulate the decision - making the capability of human experts and usually used in medical fields and in diagnostics systems. This is done with the use of the IF THEN - ELSE statements or rules. Fuzzy logic and expert systems or knowledge - based systems are two of the branches of Artificial Intelligence that use these statements. Expert systems are applications that solve problems with a set of rules or also known as the knowledge base in the form of a series of questions. From these, the expert system can diagnose and interpret the inputs from the user [4]. Fuzzy logic, on the other hand, uses membership functions in diagnosing and interpreting inputs from the user. It allows the user to have an input based on degrees of truth rather than Boolean logic inputs or crisp values [5].

The second goal of Artificial Intelligence is achieved through machine learning. This refers to the automated detection of meaningful patterns in data like in pattern recognition [6]. It is usually done by artificial neural networks wherein it emulates the structure of the nerve cells by having different layers with different neurons that have the capability to learn.

Fuzzy logic itself or expert system or neural network are common algorithms used in the medical field. There are existing studies conducted that used one or a combination of these systems. The combination of these three resulted in a hybrid system that diagnoses different diseases. But the problem with these studies is its 
availability because its main users are not the patient itself but the medical practitioners or medical personnel. It also uses a desktop platform which makes it not convenient for the common people who does not have computers or the internet. Therefore, this research is a hybrid intelligent system engineered by a neural network, fuzzy logic and expert system techniques for the diagnosis of the diseases associated with fever. It is a mobile-based application that aims at providing an efficient decision support platform to aid people with fever in diagnosing their disease and whether or not to seek medical attention especially in developing countries like the Philippines. It diagnoses common health problems experienced by Filipinos based on the Philippine Health Advisories such as influenza, dengue, chicken pox, malaria, typhoid fever, measles, hepatitis A and pneumonia [7].

The following are the objectives of the application system:

1. To establish the knowledge of the expert in the system given the levels of symptoms from the user

2. To develop the architecture of the mobile-based system using the Neuro-Fuzzy inference engine with the Takagi-Sugeno method

3. To optimize the parameters of the inference engine using neural network

This research paper is divided into five parts. The first part is the introductory section where it discusses the current problem and the overview of the study while the second part is the related works where it discusses the current technologies about the subject matter and its difference with this research. The third part is the research methodology where it discusses how the study is conducted and developed. The results of the testing done by the researchers and the medical consultants are discussed in the fourth part and the last part is the conclusion based on findings from the system implementation.

\section{RELATED WORKS}

There are various researches and studies in diagnosing an illness, with the use of neural networks, fuzzy logic and/or expert system. These studies diagnosed different diseases with different platform and techniques with different accuracy result. [8] designed a web-based expert multi-fever diagnosis system using a novel fuzzy symptom classifier with human self-observed physiological symptoms. It diagnoses common types of fever widely reported with scientifically available medication which includes malaria, dengue, typhoid, Lassa and yellow fever with the use of Fuzzy Logic. The system has two levels of diagnosis: determining the type of fever and ascertaining the level of infection as either mild or severe. However, there must be an internet connection to use this system which makes it difficult for people who do not have an internet connection. Furthermore, the levels or degree of the symptoms is not considered which might be of important information for other diseases associated with fever. There are other web systems developed with the same technique. In 2013, [9] developed a web-based application which diagnoses multi-fever like Malaria, Dengue, Typhoid, Lassa, Hay, Peeebstein, Leptospirosis and Rheumatic fever using the simple if-then-rules technique. The user interface is good, however, it is prone to error due to the lack of the various types of fever based on symptoms not defined in the ifthen-rules of the system as some might lead to other fever types. Moreover, the login function of the system might confuse the users because it seems that before one could use the system, he/she must register first. Considering this function, its sole purpose is only for the identification of the patient, not as part of the patient history or part of the calculation of the patient's diagnosis. [5] designed a web system that diagnoses human disease using a fuzzy expert system. This system focuses on the diseases in the different part of the body and its error rate or "confidence level" in every disease is greater than one. This may be because of the catalyst factor given to each disease.

In addition, there are other studies conducted with a different platform. [10] and [11] developed systems that diagnose Typhoid Fever. These are an advancement on the performance of typhoid fever diagnosis systems based on neuro-fuzzy, but its applicability on other fever types are not considered, as well as symptoms interrelationship among different fever types are not also considered in the neuro-fuzzy system. Both studies are also tried and tested with Matlab software hence, these are not available for a wide range of users. [11] system uses a genetic algorithm to enhance the optimum performance of neuro-fuzzy systems but it has the accuracy rate of $92.73 \%$ giving an error rate of $7.27 \%$. In 2012, a system [12] which diagnoses the risk in dengue patients is developed. It uses Adaptive Neuro-Fuzzy inference engine and neural network for the optimization of parameters to the inference engine. However, it only focuses on dengue fever and has an accuracy rate of $86.13 \%$. The system needs to be enhanced to achieve the error-free system since it has to do with human life. Another system developed in the different platform is the work of [13]. They developed a mobile-based system that diagnoses Malaria using Fuzzy Expert System. The system output's a percentage which might be confusing for the user. Moreover, the system works in mobile devices with supported MIDP 2.0 - CLDC 1.0, in other words, this would not work with devices running Android OS considering that most phones now run in this OS.

Therefore, MeDevice is an application system that uses a method that promises to optimize the accuracy of the diagnosis of the disease associated with fever through Backpropagation neural network and fuzzy expert algorithms and makes it available to mobile users' specifically Android devices.

\section{RESEARCH METHODOLOGY}

MeDevice is a mobile-based application that runs on Android devices and serves as a decision support system 
for its users. The application runs in Android devices with a minimum OS of Android 4.4.2 version. Furthermore, the application's GUI suits better in $3 \times 5$ inch screen though it is also working in larger screens. The user inputs the level of his/her symptoms and the application displays the diagnosed disease of the patient together with its details and the first aid treatment. The disease is based on the mentioned diseases associated with fever in the Philippine Health Advisory. The overall process of the MeDevice application is shown in Fig 1.

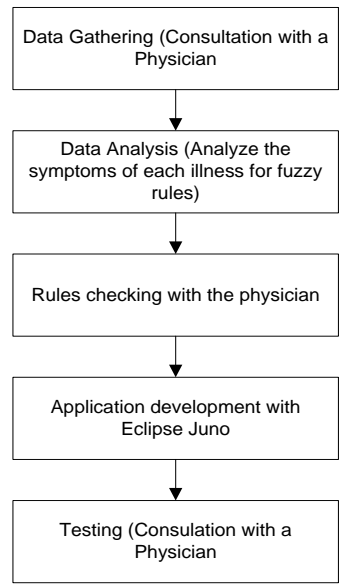

Fig.1. Conceptual Framework of MeDevice Application

\section{A. Expert System and Fuzzy Logic}

This study uses the combination of the expert system and fuzzy logic in processing the inputs from the user. It allows the user to select the symptoms and the level of the symptoms that he/she feels. Expert systems are the applications or computer programs that can mimic experts knowledge in a given problem domain. It usually answers in Boolean logic, either true or false or yes or no [5].

Fuzzy logic, on the other hand, allows its user to have a multi-valued inputs instead of 2 inputs only. It is developed by Lotfi Zadeh and introduced the concept of the fuzzy sets with its degrees of membership [14].

This study has 4 fuzzy sets for each symptom: low, mild, high and severe and uses Triangular membership function for the computation of its membership degrees. The membership function is thus given by

$$
\mu_{i j}=\left\{\begin{array}{rl}
0 & x \leq p 1 \\
\frac{x-p 1}{a-p 1} & p 1<x \leq a \\
\frac{p 2-x}{p 2-a} & a<x<p 2 \\
0 & x \geq p 2
\end{array}\right.
$$

where $\mu_{\mathrm{ij}}$ is the degree of membership function, $i$ is the number of inputs, $j$ is the fuzzy sets, $x$ is the input symptom and $p 1, p 2$ and $a$ are all parameters set.
The fuzzy rule base of the study is characterized by the sets of if-then - rules. These rules apply the Takagi Sugeno Inference systems (IS) where the premise is the description of the fuzzy subspace and its consequence is a linear input-output relation [15]. This inference system works at its full capacity in real-time applications [16]. The inference system consists of a set of $m$ fuzzy rules of the form

Rule $m$ :

$$
\text { If } x_{1} \text { is } A_{l} \text { and ... and } x_{n} \text { is } A_{n} \text { then } w_{m} \text { is } W_{d j}
$$

W

here $x_{l} \ldots . x_{n}$ are the input symptoms, $m$ is the rule number, $A_{l} \ldots A_{n}$ is the linguistic values of the rule - antecedent, $W_{d j}$ is the rule output in relation to rule $m, d$ is the diagnosed disease and $j$ is the fuzzy set. The output of each rule is a singleton set defined as a real number $w_{m}$. Using the Center of Gravity defuzzification method, the control output from the rule set is calculated as

$$
y^{\prime}=\frac{\sum_{i=1}^{m} \boldsymbol{r}_{i} \boldsymbol{w}_{i}}{\sum_{i=1}^{m} \boldsymbol{r}_{i}}
$$

where $y^{\prime}$ is the output of the fuzzy inference engine, $r_{i}$ is the product of all inputs defined in every rule, $w_{i}$ is the output of each rule and $m$ is the number of rules defined in the knowledge base.

\section{B. Neuro-Fuzzy Approach}

Fuzzy expert systems have been widely used in decision-making systems because of its capability to emulate expert knowledge, it still needs great learning for it to be precise with its decision. It cannot fully acquire the knowledge given the limited number of rules, hence it is important to provide a learning capability for these systems [17].

An artificial neural network is an architecture where it mimics the structure of the human brain by having a series of layers and neurons for each layer. This study uses the Back Propagation Neural Network algorithm for its neuro-fuzzy approach and the Gradient Descent optimization algorithm to minimize the error result of the application without changing the fuzzy rules. The theoretical framework of the Neuro-Fuzzy Approach is shown in fig 2 below

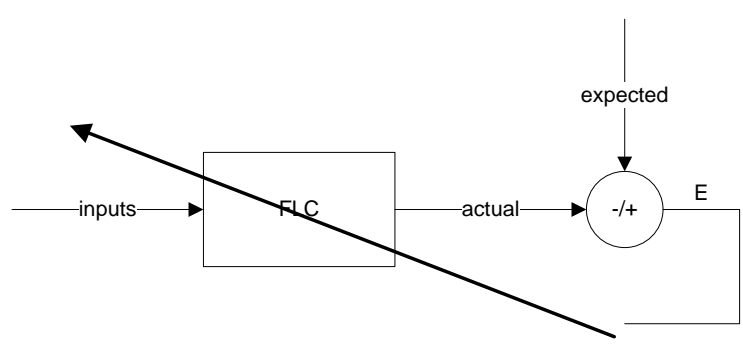

Fig.2. Architectural Framework of Neuro-Fuzzy Approach 
where $F L C$ is the fuzzy logic controller, the inputs in the FLC are the symptoms, the actual is the actual output of the FLC, expected is the expected output corresponding to the input symptom and $E$ is the error percentage or the cost function. The cost function $E$ is calculated as

$$
E=\frac{\left(y-y^{\prime}\right)^{2}}{2}
$$

where $y^{\prime}$ is the defuzzification output in (2) and $y$ is the expected output given the input symptoms.

The steepest gradient descent algorithm seeks to decrease the value of the cost function $E$ in every iteration of the network. This is done by updating the values of $a$ (center of the fuzzy set) in (1), $b$ (width of the fuzzy set) and $w_{m}$ for every fuzzy rule. Optimizing these parameter values give the following iterative equations

$$
\begin{gathered}
\boldsymbol{W}_{m}{ }^{(t+1)}=\boldsymbol{W}_{m}(t)-\gamma \frac{\partial E}{\partial \boldsymbol{W}_{m}(t)} \\
\boldsymbol{a}_{n j}(t+1)=\boldsymbol{a}_{n j}(t)-\alpha \frac{\partial E}{\boldsymbol{a}_{n j}(t)} \\
\boldsymbol{b}_{n j}{ }^{(t+1)}=\boldsymbol{b}_{n j}(t)-\beta \frac{\partial E}{\boldsymbol{b}_{n j}(t)}
\end{gathered}
$$

where $w_{m}(t+1), a_{n j}(t+1)$ and $b_{n j}(t+1)$ are the new values of the parameters and $\gamma, \alpha$ and $\beta$ are all learning rate which determines the size of the adjustments made. Here $n$ refers to the number of input, $j$ refers to the number of fuzzy sets and $m$ refers to the number of rules. The learning rates have a static value.

Fig. 3 shows the block diagram of the application system.

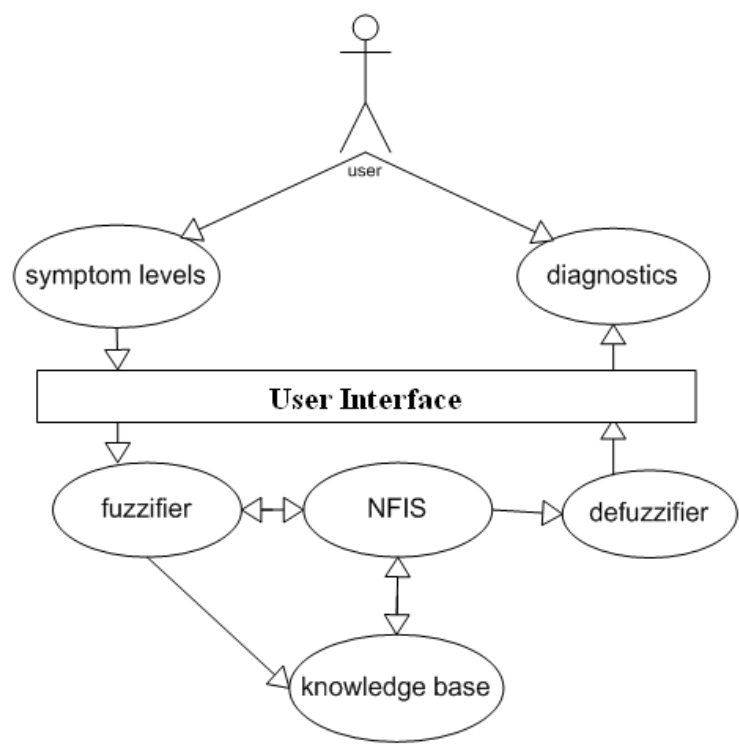

Fig.3. Block diagram of the application

\section{RESULTS AND DISCUSSION}

MeDevice is developed using Eclipse Juno IDE for the Android application. However, it uses Textpad editor for the training since it needs more memory and faster processing for its iteration.

The cost function $E$ has successfully decreased in every iteration or epoch. Figure 4 shows the values of the cost function $E$ in every iteration $e$.

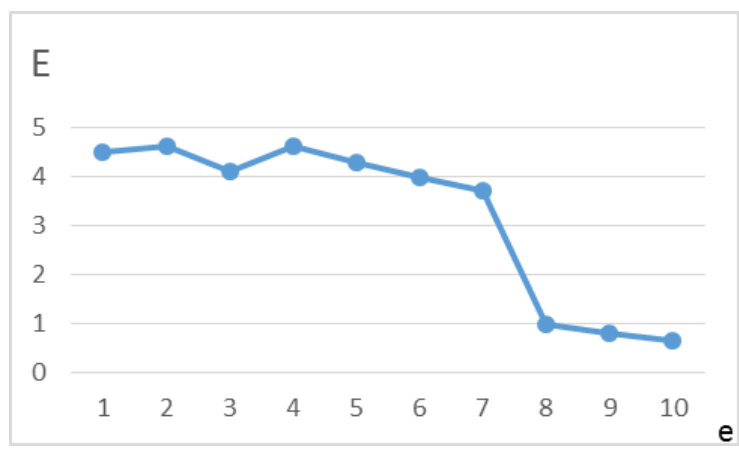

Fig.4. Cost Function $E$ values after every iteration e

The fuzzy sets also change after the learning algorithm is done as shown in fig 5 .

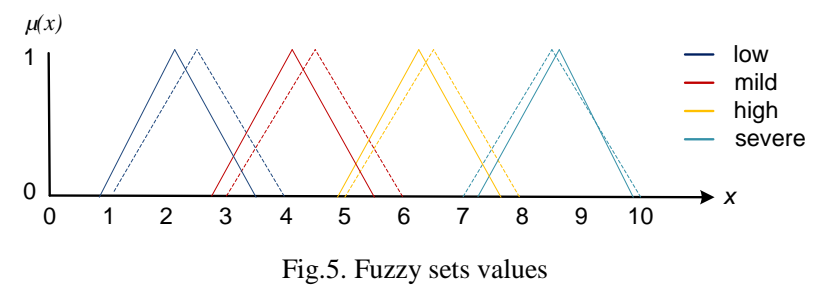

Here, the straight lines display the new values of the fuzzy sets while the dotted lines corresponds to the original values of the fuzzy sets after the learning process.

It is also noticed that the values of the learning rates $\gamma$, $\alpha$ and $\beta$ have a great impact on the result. If its values are too small or too big, the cost function increases gradually. The learning rate is one of the values that must be adjusted properly during the training to achieve the optimum value of the cost function $E$.

Table 1 shows the result of the testing both for the testing data set and the actual testing of the physicians. There are 66 records of given symptoms and its levels with its illness have been used for its testing and 50 patients data for the actual testing conducted by physicians. There are 5 physicians and they tried to test 10 records each.

Table 1. Accuracy result of the application

\begin{tabular}{|c|c|c|}
\hline & No. of Patients/Data & Result \\
\hline Testing Dataset & 66 & $100 \%$ \\
\hline Physicians & 50 & $98 \%$ \\
\hline
\end{tabular}

The application has an accuracy result of $98 \%$ of the actual testing of the physicians. This is because they got confused about the symptoms listed in the application and. 
its levels. Furthermore, they are still looking for more other information like the result of the laboratory tests But, in general, they still agree with the application's result. The objectives of the application have been met also. Fig. 6 to fig. 9 show the user interface of the application.

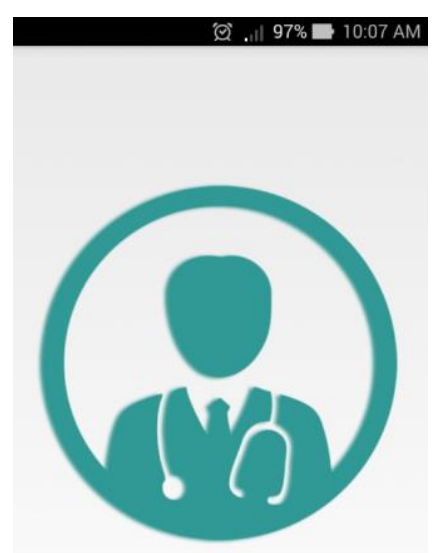

MeDevice

Fig.6. Splash screen of the application

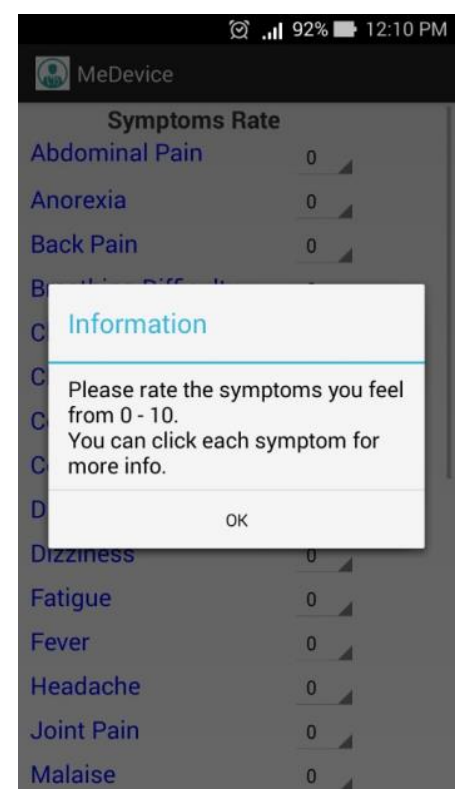

Fig.7. Symptoms Interface of the Application

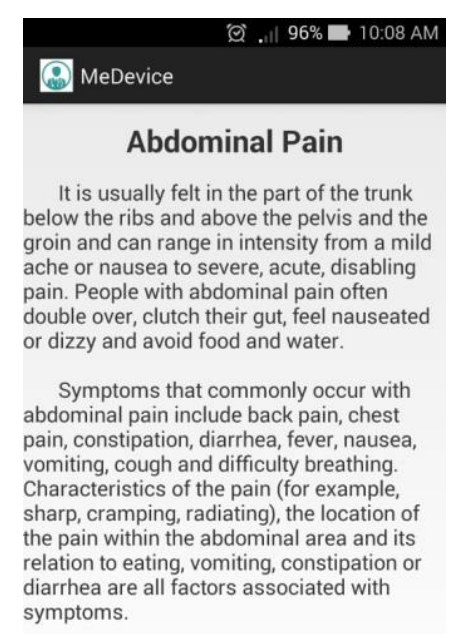

Fig.8. Symptom details of the application

MeDevice
Influenza
Treatment
- Use of antiviral agents, such as
oseltamivir (Tamiflu) or zanamivir (Relenza),
within the first 2 days may shorten the
illness and help prevent serious
complications.
- Have adequate rest.
- Increase intake of oral fluids and
nutritious foods.
- Take paracetamol for fever. Aspirin should
not be given to children.
- Antibiotics should be given only to
complications of influenza, such as
pneumonia or otitis media.
Prevention
- Give influenza vaccination preferably
annually.
- Minimize contact with a person who has
influenza.
- Avoid crowded places.
- Distance yourself by at least 1 meter from

Fig.9. Diagnostic output of the application

\section{CONCLUSION}

MeDevice is a mobile application that processes the input symptoms and diagnoses what illness it is such as pneumonia, dengue, malaria, hepatitis A, chicken pox, typhoid fever, influenza or measles. It is also noted that the main symptom of these illnesses is a fever. Furthermore it emulates the experts' knowledge through the rules defined and the fuzzy inference engine. 
MeDevice proves that Takagi - Sugeno Inference System can also be used in decision - support applications. Moreover, with the help of the optimization algorithm and back propagation neural network, it helps the inference system to learn on its own to achieve the optimum value of the cost function. With the right value of the learning rate, this helps the application to achieve high accuracy result both from the testing data and the physicians testing. It is suggested that for the future study, more diseases must be included in the application

\section{ACKNOWLEDGMENT}

The authors would like to thank everyone who helped us in making this study possible especially to Dr. Nick Pabiona as our major consultant and to Notre Dame University.

\section{REFERENCES}

[1] D. Ogoina, "Fever, fever patterns and diseases called 'Fever' - A Review" J Infect Public Health, vol 4(3), pp. 108-24, August 2011, doi: 10.1016/j.jiph.2011.05.002.

[2] J. Taber, Ph.D., B. Leyva, B.A. and A. Persoskie, A, "Why do People Avoid Medical Care? A Qualitative Study using National Data", J Gen Intern Med, vol 30(3), pp. 290-297, March 2015, doi: 10.1007/s11606-014-30891

[3] V. Makoge, H. Maat, L. Vaandrager and M. Koelen, "Health-Seeking Behaviour towards Poverty-Related Disease (PRDs): A Qualitative Study of People Living in Camps and on Campuses in Cameroon", PLoS Negl Trop Dis, Vol 11(1). January 2017, doi: 10.1371/journal.pntd.0005218

[4] Tutorials Point, "Artificial Intelligence" [Online], 2015 Available: http://www.tutorialspoint.com

[5] M. Hasan, K. Sher-E-Alam and A. Chowdhury, "Human Disease Diagnosis using a Fuzzy Expert System", Journal of Computing, vol 2(6), June 2010, pp. 66

[6] S. Shwartz and S. David, Understanding Machine Learning: From Theory to Algorithms, Cambridge University Press, 2014

[7] Department of Health, Philippine Health Advisories, National Center for Health Promotion, 2012

[8] I.B. Ajenaghughurure and P. Sujatha Ph.D., "Fuzzy Based Multi-Fever Symptom Classifier Diagnosis Model", I. J. Information Technology and Computer Science, vol 10(13), 2017, pp. 13-28, doi: 10.5815/ijitcs.2017.10.02

[9] S. Tunmibi, O. Adeniji, A. Aregbesola and A. Dasylva, "A Rule Based Expert System for Diagnosis of Fever", Int J Adv Res, vol 1(7), September 2017, pp. 343-348

[10] O. W. Samuel and M.O. Omisorre, "Hybrid Intelligent System for the Diagnosis of Typhoid Fever", J Comput Eng Inf Technol, vol2(2), August 2013, doi: 10.4172/2324-9307.1000109

[11] M.G. Asogbon, O.W. Samuel, M.O. Omisorre and O. Awonusi, "Enhanced Neuro-Fuzzy System Based on Genetic Algorithm for Medical Diagnosis", J Med Diagn Methods, vol 5(1), February 2016, doi: 10.4172/21689784.1000205

[12] T. Faisal, M.N. Taib and F. Ibrahim, "Adaptive NeuroFuzzy Inference System for Diagnosis Risk in Dengue Patients", Expert Syst Appl, vol 39(4), March 2012, pp. 4483-4495, https://doi.org/10.1016/j.eswa.2011.09.140

[13] A. Owoseni and I. Ogundahunsi, "Mobile-Based Fuzzy
Expert System for Diagnosing Malaria”, I J Information Engineering and Electronnic Business, vol 2, March 2016 , pp. 14-22, doi: 10.5815/ijieeb.2016.02.02

[14] L.A. Zadeh, "Fuzzy Sets versus Probability", Proceeding of the IEEE. Vol 68(3). March 1980, pp. 421 - 421. Doi: 10.1109/PROC.1980.11659

[15] T. Takagi and M. Sugeno, "Fuzzy Identification of Systems and Its Applications to Modeling and Control", IEEE Trans Cybern, Vol SMC-15(1), January 1985, Doi: 10.1109/TSMC.1985.6313399

[16] M. Blej, and M. Azizi, "Comparison of Mamdani - Type and Sugeno - Type Fuzzy Inference Systems for Fuzzy Real Time Scheduling”, Int J Appl Eng Res, vol 11(22), 2016, pp. 11071-11075

[17] A. Lotfi, "The Importance of Learning in Fuzzy Systems", European Society for Fuzzy Logic and Technology. (Online) Available: http://www.academia.edu/download/5589029/10.1.1.145. 366.pdf

\section{Authors' Profiles}

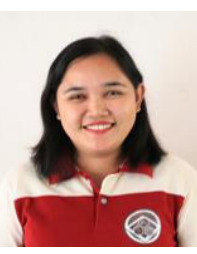

Johaira U. Lidasan is a faculty of College of Computer Studies in Notre Dame University, Philippines. She is a graduate of Master in Computer Science and a certified Microsoft Office Specialist and IBM DB2 Academic Associate. She is a member of Computing Society of the Philippines and she is interested in image processing, fuzzy logic, expert system and neural networks.

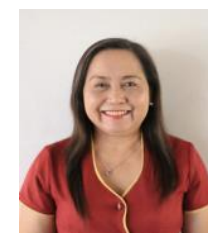

Martina P. Tagacay is the college dean of the College of Computer Studies in Notre Dame University, Philippines and an officer of the Philippine Society of Information Technology Educators. She is a graduate of Master in Business Administration and Master in Information Management. She is interested in data mining.

How to cite this paper: Johaira U. Lidasan, Martina P. Tagacay, "MeDevice: A Mobile - Based Diagnosis of Common Human Illnesses using Neuro - Fuzzy Expert System", International Journal of Information Technology and Computer Science(IJITCS), Vol.11, No.8, pp.27-32, 2019. DOI: 10.5815/ijitcs.2019.08.04 